\title{
ASPECTOS CLÍNICOS E PATOLÓGICOS EM FRANGOS DE CORTE (Gallus gallus domesticus) INFECTADOS EXPERIMENTALMENTE COM OOCISTOS ESPORULADOS DE Eimeria acervulina TYZZER, 1929
}

\author{
FAGNER LUIZ DA C. FREITAS ${ }^{1}$, KATYANE DE S. ALMEIDA², ADJAIR A. DO NASCIMENTO², \\ JOSÉ H. TEBALDI' ${ }^{2}$, ROSANGELA Z. MACHADO ${ }^{1}$; CELIO R. MACHADO ${ }^{3}$
}

\begin{abstract}
FREITAS, F.L. DA C.; ALMEIDA, K. DE S.; NASCIMENTO, A.A. DO; TEBALDI, J.H.; MACHADO, R.Z.; MACHADO, C.R. [Clinical and pathological aspects in broilers (Gallus gallus domesticus) infected experimentally with Eimeria acervulina Tyzzer, 1929 sporulated oocysts]. Aspectos clínicos e patológicos em frangos de corte (Gallus gallus domesticus) infectados experimentalmente com oocistos esporulados de Eimeria acervulina Tyzzer, 1929. Revista Brasileira de Parasitologia Veterinária, v. 17, n. 1, p.16-20, 2008. Programa de Pós-Graduação em Medicina Veterinária, Departamento de Patologia Animal, Universidade Estadual Paulista, Campus de Jaboticabal, Av. Prof. Paulo Donato Castellane, s/n, Zona Rural, Jaboticabal, SP 14884-900, Brazil.E-mail: fagner@fcav.unesp.br.

In the present study, clinical signs and pathological changes were evaluated during 30 days in broilers, lineage Cobb, males, with ten days old, infected with Eimeria acervulina. Were used 192 animals distributed in 3 groups: group A inoculated with $1 \times 10^{6}$ sporulated oocysts; group B inoculated with $1 \times 10^{5}$ sporulated oocysts; group C inoculated with distilled water. The clinical signs observed were anorexia, diarrhea and apathy. The pathological macroscopic changes observed were: enteritis, hyperemia with subsequent intestinal congestion, high concentration of mucus exudates in the lumen of the small intestine, pale and dehydration muscle, gall bladder full of liquid and deposition of hepatic fat. The villous atrophy and high presence of inflammatory cells were the microscopic changes observed in the gut epithelium. In histopathologic analysis of liver observed inflammatory cells infiltrated and fat deposition. The results demonstrate that broilers experimentally infected with E. acervulina presented progressive intestinal lesions of variable intensity and that these abnormalities are the main cause of reduction of bird performance.
\end{abstract}

KEY WORDS: Eimeria acervulina, experimentally infection and broilers.

\section{RESUMO}

No presente estudo, sinais clínicos e alterações patológicas foram avaliados por 30 dias em frangos de corte, linhagem Cobb, machos, com dez dias de idade, infectados com Eimeria acervulina. Foram utilizados 192 animais distribuídos em 3 grupos: grupo A inoculado com $1 \times 10^{6}$ oocistos esporulados; grupo B inoculado com $1 \times 10^{5}$ oocistos esporulados; grupo C inoculado com água destilada. Os sinais clínicos observados foram anorexia, diarréia e apatia. As alterações patológicas macroscópicas observadas foram: enterite, hiperemia seguido de congestão intesti-

\footnotetext{
${ }^{1}$ Departamento de Patologia Animal, Universidade Estadual Paulista (Unesp), Campus de Jaboticabal, São Paulo, Via de acesso Prof. Paulo Donato Castellane, s/n, Jaboticabal, SP 14884-900, Brasil. E-mail: fagner@fcav.unesp.br

2 Departamento de Medicina Veterinária Preventiva, Unesp-Campus de Jaboticabal, SP, Brasil.

${ }^{3}$ Departamento de Morfologia e Fisiologia Animal, Unesp-Campus de Jaboticabal, SP, Brasil.
}

nal, excesso de exsudato mucoso no lúmen do intestino delgado, palidez e desidratação muscular, alto acúmulo de bile na vesícula biliar e deposição de gordura hepática. A atrofia de vilosidades e alta presença de células inflamatórias foram as alterações microscópicas observadas no epitélio intestinal. Na análise histopatológica do fígado observaram-se infiltrados inflamatórios e deposição de gordura. Os resultados demonstraram que frangos de corte infectados experimentalmente com E. acervulina apresentam progressivas lesões intestinais de intensidade variável e que essas anormalidades são as principais causas de redução no desenvolvimento da ave.

PALAVRAS-CHAVE: Eimeria acervulina, infecção experimental e frangos de corte.

\section{INTRODUÇÃO}

Dentre as doenças que acarretam consideráveis perdas econômicas destaca-se a eimeriose, enfermidade mais freqüen- 
te na avicultura industrial mundial (WILIANMS, 1999), causada por diferentes espécies do gênero Eimeria. Dentre as espécies, destaca-se a E. acervulina que, segundo Morehouse e Mcguire (1958), apresenta-se, freqüentemente, na forma subclínica ocasionando prejuízos na conversão alimentar e diminuição no crescimento e ganho de peso das aves. No Brasil, o primeiro caso de eimeriose aviária foi relatado por Reis e Nóbrega (1936) no Estado de São Paulo e dentre as espécies descritas encontrava-se E. acervulina.

Segundo Dickison (1941), E. acervulina apresenta um período pré-patente de 96 horas e um alto grau de morbidade devido ao seu elevado potencial reprodutivo, sendo a espécie que produz maior quantidade de oocistos. De acordo com Morehouse e Mcguire (1958), o parasita invade as células epiteliais do duodeno, onde a infecção é mais severa, e avança até a parte média do intestino delgado causando destruição epitelial e conseqüente enterite. Segundo Terra et al. (2001) as lesões provocadas no epitélio intestinal, resultantes das fases de desenvolvimento, prejudicam os índices zootécnicos dos frangos e, conseqüentemente, o resultado econômico da empresa avícola.

Os mecanismos envolvidos nessas alterações não estão totalmente esclarecidos quanto à época pós-infecção em que ocorrem e a repercussão desta infecção no ganho de peso do hospedeiro, tendo apenas estudos realizados em décadas anteriores utilizando-se linhagens de aves com baixo desempenho zootécnico quando comparadas com as linhagens de crescimento rápido utilizadas atualmente. O presente trabalho tem como objetivo avaliar os períodos parasitários, sinais clínicos e alterações patológicas de frangos infectados experimentalmente com E. acervulina.

\section{MATERIAL E MÉTODOS}

Foram utilizados cento e noventa e dois frangos de corte, linhagem Cobb, machos, com dez dias de idade, provenientes do incubatório da empresa Frango Sertanejo S.A localizado em Guapiaçú, SP. As aves foram randomizadas de acordo com o peso e distribuídos em três grupos experimentais: grupo A, inoculado com $1 \mathrm{ml}$ de solução contendo $1 \times 10^{6}$ oocistos esporulados de E. acervulina; grupo B, inoculado com $1 \mathrm{ml}$ de solução contendo $1 \times 10^{5}$ oocistos esporulados de $E$. acervulina; animais pertencentes ao grupo $\mathrm{C}$ foram utilizados como controles, sendo inoculado com $1 \mathrm{ml}$ de água destilada. A dose escolhida para o inóculo foi baseada no guia de avaliação de drogas anticoccidianas elaborado por Holdsworth et al. (2004). Para a inoculação, todas as aves do experimento foram contidas manualmente e inoculadas, com o auxílio de uma pipeta automática, por via oral.

Cada grupo foi instalado em gaiólas de ferro com dimensões de $1 \mathrm{~m}$ de comprimento x $2 \mathrm{~m}$ de largura $\mathrm{x} 1,5 \mathrm{~m}$ de altura. Os comedouros e bebedouros utilizados contendo, respectivamente, água limpa e ração balanceada sem anticoccidianos, foram do tipo calha, sendo lavados com água e detergente neutro e flambados a cada 12 horas para que o risco de reinfecção fosse evitado.
A composição da ração utilizada (à base de milho e farelo de soja) obedeceu às normas e padrões nutricionais estabelecidas pela NRC (1998), sendo isonutritiva e isoenergética e fornecida às aves ad libitum.

Durante todo o período experimental, foram realizados exames clínicos nos animais e análises parasitológicas em suas fezes para detecção dos períodos de pré-patência e patência de E. acervulina, sendo utilizada a técnica de centrífugoflutuação de acordo com Hoffmann (1987).

Para observação das alterações patológicas foram abatidas, diariamente, conforme resolução da Comissão de Ética e Bem Estar Animal da FCAV/UNESP (CEBEA), duas aves por grupo para elucidação dos dias mais importantes a serem estudados com mais detalhes em futuras pesquisas. Para realização dos exames histopatológicos foram retirados fragmentos de fígado, duodeno, jejuno e íleo, sendo esses tecidos conservados em solução de formalina tamponada a $10 \%$ e, posteriormente, embebidos e incluídos em parafina, cortados no micrótomo, colocados em lâminas e corados com hematoxilina-eosina (HE).

\section{RESULTADOS}

Os primeiros sinais clínicos foram observados no $4^{\circ} \mathrm{DPI}$, sendo encontrada anorexia, seguida de um quadro de apatia e diarréia fétida associada ou não com pequenas estrias esbranquiçadas permanecendo até o $30^{\circ}$ DPI. As estrias esbranquiçadas eliminadas pelos animais devem-se à descamação do epitélio intestinal afetado por E. acervulina, sendo visualizados ao microscópio oocistos imaturos aderidos à mucosa (Figura 1). O período pré-patente observado foi de 96 horas (quatro dias) e o período patente foi de 15 dias para os dois grupos infectados, terminando no $18^{\circ}$ DPI. O término do período patente foi confirmado pelo exame parasitológico das fezes e análise histopatológica do intestino delgado durante três dias consecutivos.

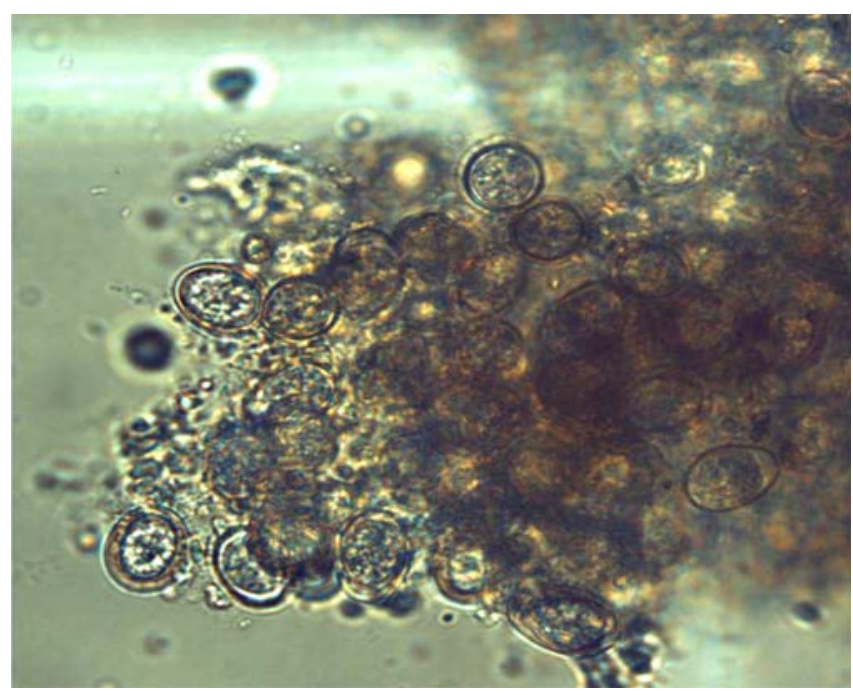

Figura 1. Fragmento de mucosa intestinal eliminado nas fezes contendo inúmeros oocistos de Eimeria acervulina em frangos inoculados com $1 \times 10^{6}$ oocistos esporulados. 400x. 


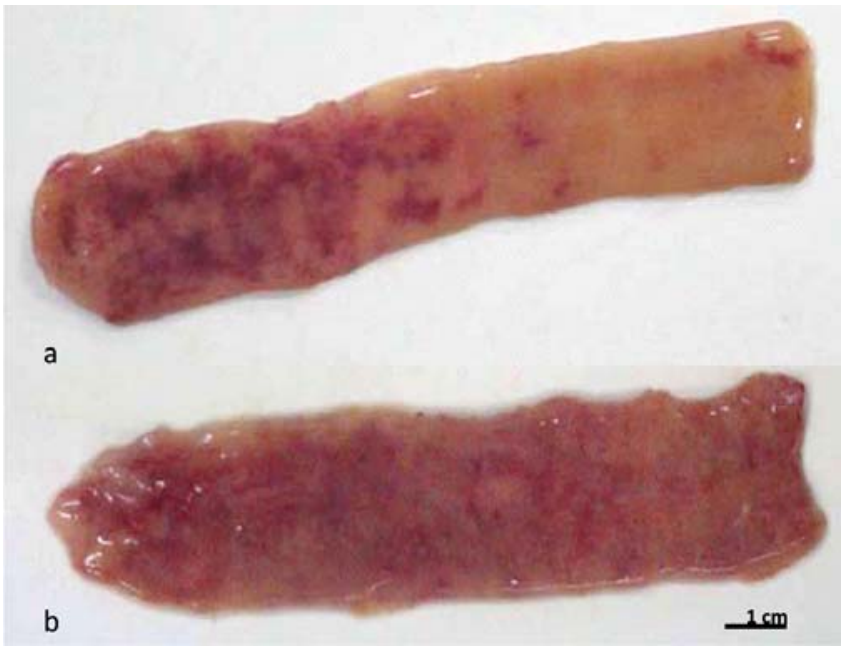

Figura 2. Áreas avermelhadas na região duodenal observada no $3^{\circ}$ (a) e $9^{\circ}$ dias após imfecção (b) em frangos inoculados com $1 \times 10^{6}$ oocistos de Eimeria acervulina.

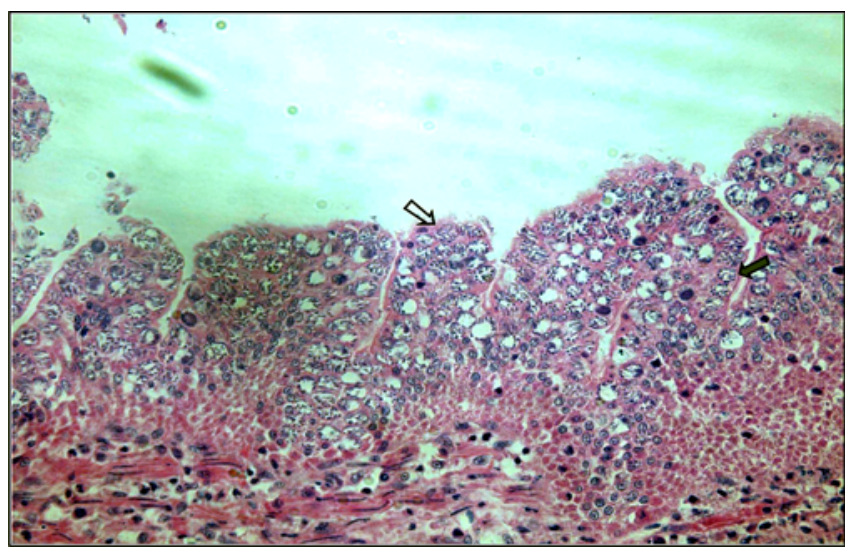

Figua 3. Atrofia de vilosidades duodenal (seta branca) associada com alta concentração de merontes (seta cinza) no $4^{\circ}$ dias após imfecção em frangos inoculados com $1 \times 10^{5}$ oocistos de Eimeria acervulina. H.E., 100x.

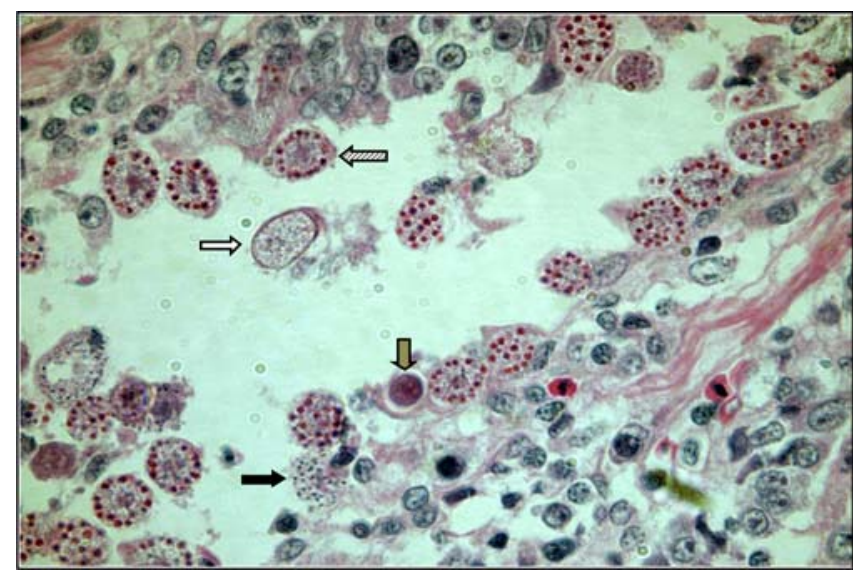

Figura 4. Oocisto (seta branca), zigoto (seta cinza), microgametócitos (seta preta) e macrogametócitos (seta listrada) na mucosa duodenal no $4^{\circ}$ dias após imfecção em frangos inoculados com $1 \times 10^{6}$ oocistos de Eimeria acervulina. H.E., 400x.
No $1^{\circ} \mathrm{DPI}$, em ambos os grupos infectados, os vasos sangüíneos da região duodenal apresentaram-se dilatados evidenciando um processo de hiperemia que rapidamente evoluiu para congestão a partir do $2^{\circ}$ DPI permanecendo até o $30^{\circ}$ DPI associado com espessamento de mucosa. No grupo controle, nenhuma alteração foi observada no duodeno, que permaneceu com aspecto normal até o $30^{\circ}$ DPI. No $2^{\circ}$ DPI, os grupos A e B apresentaram petéquias na região duodenal que evoluíram para uma lesão maior (Figura 2) estendendo-se para a região proximal do jejuno e atingindo levemente o íleo. Ao fazer uma incisão transversal no duodeno, observou-se que a região da mucosa localizada próxima ao lúmen apresentavase hemorrágica caracterizando a área afetada pelo parasito. No grupo B, também foi encontrada enterite, porém em menor intensidade, sendo evidenciados pontos de coloração creme no $6^{\circ}$ DPI que evoluíram para estrias transversais coalescentes em torno do $9^{\circ} \mathrm{DPI}$; essas estrias não foram de fácil visualização nos animais pertencentes ao grupo A devido à intensa reação inflamatória local fazendo com que houvesse uma compactação dessas estrias dificultando a sua visualização.

A partir do $5^{\circ}$ DPI, em ambos os grupos infectados, foi encontrado exsudato mucoso no lúmen do intestino delgado, principalmente na região duodenal e na parte proximal do jejuno. Também foi observado retenção de alimento no próventrículo e moela e acúmulo de bile na vesícula e palidez e desidratação muscular, sendo mais observadas nos músculos do peito e da coxa.

Os animais dos grupos infectados apresentaram fígado de coloração amarelo-pálido a partir do $8^{\circ}$ DPI permanecendo até o $30^{\circ} \mathrm{DPI}$, ocorrendo devido à deposição de gordura, levando a crer que o transporte e regulação de lipídios nesse órgão estejam afetados. Na análise histopatológica do fígado foram observados infiltrados inflamatórios multifocais e gotículas de lipídios intracitoplasmáticas.

As análises histopatológicas dos segmentos intestinais, principalmente do duodeno, caracterizaram-se por intensos infiltrados inflamatórios no epitélio intestinal, associado com descamação e atrofia de vilosidade (Figura 3). A enterite severa visualizada macroscopicamente também foi observada no exame histopatológico. Quanto às estruturas parasitárias, no $4^{\circ}$ DPI já era possível observar grande quantidade de merontes na mucosa duodenal, com uma quantidade menor no jejuno, localizando-se principalmente nas criptas do epitélio. Neste trabalho também foi possível observar grande quantidade de macrogamontes e microgamontes, assim como zigotos e oocistos recém formados (Figura 4).

\section{DISCUSSÃO}

O quadro clínico de anorexia, diarréia e apatia foi diretamente proporcional à dose infectante, sendo conseqüência primária da enterite resultante do desenvolvimento do parasito. As alterações clínicas encontradas no presente trabalho são semelhantes às alterações observadas por Long (1968), 
Michael e Hodges (1971) e Kessler et al. (1979) e podem passar despercebidas nas granjas avícolas, tendo-se em vista o grande número de animais, o que agrava ainda mais o problema.

O aparecimento de petéquias e depois uma severa enterite, também foi resultante do ciclo reprodutivo do parasito, principalmente devido à liberação de merozoítos (pelos merontes rompidos) e oocistos da mucosa.

Michael e Hodges (1971) estudando frangos infectados com $1 \times 10^{6}$ de oocistos de E. acervulina observaram espessamento de mucosa no intestino, particularmente no duodeno e extensa inflamação na região duodenal no $3^{\circ}$ DPI características de uma enterite severa. Morehouse e Mcguire (1958) observaram uma severa reação inflamatória na mucosa intestinal inoculando aves com $20 \times 10^{6}$ oocistos de $E$. acervulina. De acordo com Jones et al. (2000), a dilatação vascular é decorrente da ação de mediadores químicos que relaxam a musculatura lisa do capilar para que as células do sistema imunológico possam eliminar o agente infeccioso. $\mathrm{O}$ sistema vascular do intestino delgado, principalmente na região do duodeno e jejuno, é de grande importância, pois tem a função de manter o gradiente de concentração durante o processo de absorção de nutrientes (VIEIRA, 2002).

Michael e Hodges (1971), observaram em frangos infectados com $1 \times 10^{6}$ oocistos de E. acervulina discretas lesões transversais esbranquiçadas na mucosa do duodeno e jejuno. Segundo Kawazoe (2000), as estrias são facilmente observadas em infecções leves e apresentam grandes quantidades de estruturas parasitárias em diferentes fases do ciclo evolutivo. A localização das estruturas parasitárias de $E$. acervulina no presente estudo são semelhantes às encontradas por Doran (1966) e Kawazoe et al. (2005).

A grande quantidade de exsudato mucoso na região anterior do intestino delgado ocorreu devido à preferência tecidual do parasito por esse local, sendo a área mais lesionada pela infecção. Esse exsudato mucoso é sintetizado e liberado pelos enterócitos e células caliciformes com objetivo de proteger a mucosa intestinal contra a ação de patógenos e do baixo pH do bolo alimentar oriundo da moela, além de auxiliar no processo de reconstituição celular nas áreas afetadas após eliminação do agente infeccioso (MAIORKA et al., 2002). Michael e Hodges (1971), observaram exsudato mucoso de coloração creme em frangos infectados com $1 \times 10^{6}$ e $20 \times 10^{6}$ oocistos de E. acervulina encontrando ainda muco de coloração esverdeada, devido ao excesso de bile, em frangos infectados com quatro doses consecutivas de $5 \times 10^{6}$ oocistos.

A retenção de alimento no pró-ventrículo e ventrículo e o freqüente encontro da vesícula biliar cheia sugerem anormalidade na ingestão e no fluxo gastrointestinal de alimento decorrentes do parasitismo, porém seu mecanismo ainda não está elucidado. A palidez e desidratação muscular foram também observadas por Becker (1940) e Morehouse e Mcguire (1958) que os atribuiu à ingestão reduzida de alimento e água.

Uma alteração importante encontrada nos animais infectados do presente trabalho foi a deposição de gordura hepática. $\mathrm{O}$ aspecto amarelado associado com áreas de inflamação causa má aparência da víscera, prejudicando assim a sua comercialização. Isso ocorreu, possivelmente, devido a uma alteração no metabolismo de lipídio hepático decorrente das lesões intestinais resultantes do ciclo evolutivo do parasito.

Todas as lesões decorrentes da reprodução parasitária interferem diretamente a absorção de nutrientes, desencadeando uma série de distúrbios no metabolismo de carboidratos (FREEMAN, 1970; ALLEN; MCMURTRY, 1984), proteínas (RUFF et al., 1981; FETTERER; ALLEN, 2000), lipídeos (ALLEN, 1988) e macro e micro-minerais (TURK, 1986ab).

Na presente pesquisa, as doses de $1 \times 10^{6} \mathrm{e} 1 \times 10^{5}$ oocistos de E. acervulina inoculadas nos grupos A e B, respectivamente, foram suficientes para ocasionar sérios danos ao organismo animal, porém o grupo A foi o mais afetado pela infecção, ficando evidente que a intensidade das alterações clinicas e patológicas é decorrente da dose infectante. Os órgãos afetados por E. acervulina são de suma importância para o processo de absorção e digestão da ave comprometendo o ganho de peso, principal critério utilizado no controle de qualidade do desempenho das aves em granjas avícolas, repercutindo em grandes perdas econômicas ao criador de frangos de corte.

\section{REFERÊNCIAS BIBLIOGRÁFICAS}

ALLEN, P.C. The effect of Eimeria acervulina infection on plasma lipids and lipoproteins in young broiler chicks. Veterinary Parasitology, v. 30, n. 1, p.17-30, 1988.

ALLEN, P.C.; MCMURTRY, J.P. Changes in pancreatic hormones associated with coccidiosis. Poultry Science, v. 63, n. 6, p.1129-1135, 1984.

BECKER, E.R. Coccidiosis of domesticated birds with special reference to the common fowl. Veterinary Medicine, v. 35, n. 1, p.401-407, 1940.

DICKINSON, E.M. The effects of variable dosages of sporulated Eimeria acervulina oocysts on chickens. Poultry Science, v. 20, n. 1, p.413-424, 1941.

DORAN, D.J. The migration of Eimeria acervulina sporozoites to the duodenal glands of Lieberkühn. Journal of Protozoology, v. 13, n. 1, p.27-33, 1966.

FETTERER, R.H.; ALLEN, P.C. Eimeria acervulina infection elevates plasma and muscle 3-methylhistidine levels in chickens. Journal of Parasitology, v. 86, n. 4, p.783-791, 2000.

FREEMAN, B.M. Carbohydrate stores in chickens infected with Eimeria tenella. Parasitology, v. 61, n. 1, p.245-251, 1970.

HOFFMANN, R.P. Diagnóstico de Parasitismo Veterinário. Porto Alegre: Sulina, 1987. 156p.

HOLDSWORTH, P.A.; CONWAY, D.P.; MCKENZIE, M.E.; DAYTON, A.D.; CHAPMAN, H.D.; MATHIS, G.F.; SKINNER, J.T.; MUNDT, H.C.; WILLIAMS, R.B. World Association for the Advancement of Veterinary Parasitology (WAAVP) guidelines for evaluating the efficacy of 
anticoccidial drugs in chickens and turkeys. Veterinary Parasitology, v. 121, n. 1, p.189-212, 2004.

JONES, T.C.; HUNT, R.D.; KING, N.W. Patologia Veterinária. $6^{\mathrm{a}}$ ed. São Paulo: Manole, 2000. 1415p.

KAWAZOE, U. Coccidiose. In: BERCHIERI JÚNIOR, A.; MACARI, M. Doenças das aves. Campinas: FACTA, 2000. p.391-405.

KAWAZOE, U.; BORDIN, E.L.; LIMA, C.A.; DIAS, L.A.V. Characterization and histopathological observations of a selected Brazilian precocius line of Eimeria acervulina. Veterinary Parasitology, v. 131, n. 1, p.5-14, 2005.

KESSLER, R.H.; SILVA, N.R.S.; HOFFMANN, R.T.; WAGNER, E.M. ocorrência de Eimeria acervulina (TYZZER, 1929) em Gallus gallus domesticus no Rio Grande do Sul. Arquivos da Faculdade de Veterinária da Universidade Federal do Rio Grande do Sul, v. 7, n. 1, p.105-119, 1979.

LONG, P.L. The pathogenic effects of Eimeria praecox and E. acervulina in the chicken. Parasitology, v. 58, n. 3, p.691 - 700, 1968.

MAIORKA, A.; BOLELI, I C.; MACARI, M. Desenvolvimento e reparo da mucosa intestinal. In: MACARI, M.; FURLAN, R.L.; GONZALES, E. Fisiologia aviária aplicada a frangos de corte. Jaboticabal: Funep/Unesp, 2002. p.113-124.

MICHAEL, E.; HODGES, R.D. The pathogenic effects of Eimeria acervulina: a comparison of single and repated infections. Veterinary Record, v. 89, n. 1, p.329-333, 1971.

MOREHOUSE, N.F.; MCGUIRE, W.C. The pathogenicity of
Eimeria acervulina. Poultry Science. v. 37, n. 1, p.665672, 1958.

NRC (National Research Council). Nutrient Requirements of Swine. 10 ed. Washington: National Academy Press, 1998. 190p.

REIS, J.; NÓBREGA, P. Doenças das Aves. São Paulo: Instituto Biológico de São Paulo, 1936. 468p.

RUFF, M.D.; ALLEN, P.C.; CHUTE, M.B. Composition of heart, liver and skeletal muscle from broilers with coccidiosis. Poultry Science, v. 60, n. 1, p.1807-1811, 1981.

TERRA, A.T.; COSTA, P.S.; FIGUEIREDO, P.C.; CARVALHO, E.C.Q. Freqüência de espécies do gênero Eimeria em frangos de cortes abatidos industrialmente no município de Monte Alegre do Sul, Estado de São Paulo. Revista Brasileira de Parasitologia Veterinária, v. 10, n. 2, p.8790, 2001.

TURK, D.E. Macroelements in the circulation of coccidiosis infected chicks. Poultry Science, v. 65, n. 1, p.462-468, 1986a.

TURK, D.E. Microelements in the circulation of coccidiosis infected chicks. Poultry Science, v. 65, n. 11, p.2098-2103, 1986b.

VIEIRA, S.L. Carboidratos: digestão e absorção. In: MACARI, M.; FURLAN, R.L.; GONZALES, E. Fisiologia aviária aplicada a frangos de corte. Jaboticabal: Funep/ Unesp, 2002. p.125-133.

WILIANMS, R.B. A compartmentalised model for the estimation of the cost of the world's chicken production industry. International Journal for Parasitology, v. 29, n. 1, p.1209-1229, 1999.

Recebido em 10 de agosto de 2006.

Aceito para publicação em 21 de março de 2008. 